\title{
Hyponatremia in the neurosurgical patient: diagnosis and management
}

\author{
Chad D. Cole, M.Sc., Oren N. GotTfried, M.D., James K. LiU, M.D., \\ and William T. Couldwell, M.D., Ph.D. \\ Department of Neurosurgery, University of Utah School of Medicine, Salt Lake City, Utah
}

\begin{abstract}
Hyponatremia is frequently encountered in patients who have undergone neurosurgery for intracranial processes. Making an accurate diagnosis between the syndrome of inappropriate secretion of antidiuretic hormone (SIADH) and cerebral salt wasting (CSW) in patients in whom hyponatremia develops is important because treatment differs greatly between the conditions. The SIADH is a volume-expanded condition, whereas CSW is a volume-contracted state that involves renal loss of sodium. Treatment for patients with SIADH is fluid restriction and treatment for patients with CSW is generally salt and water replacement. In this review, the authors discuss the differential diagnosis of hyponatremia, distinguish SIADH from CSW, and highlight the diagnosis and management of hyponatremia, which is commonly encountered in patients who have undergone neurosurgery, specifically those with traumatic brain injury, aneurysmal subarachnoid hemorrhage, recent transsphenoidal surgery for pituitary tumors, and postoperative cranial vault reconstruction for craniosynostosis.
\end{abstract}

KEY WoRDS - hyponatremia - syndrome of inappropriate antidiuretic hormone secretion • cerebral salt wasting • intracranial disease

Hyponatremia is a common electrolyte disorder encountered in patients in the neurosurgical ICU., 1,56 The causes of hyponatremia are diverse and the associated risks of morbidity vary widely. ${ }^{5,17}$ The etiological range of this electrolyte disorder can be subcategorized into hyponatremia associated with high, normal, or low osmolality (Fig. 1).1,3 Neurological dysfunction is the principal manifestation of hyponatremia, which may be exacerbated by other disease processes or underlying conditions, especially in those patients in whom a pathological condition is located intracranially. Awareness of other potential causes of hyponatremia is required to provide appropriate management and avoid deleterious outcomes, which may occur when the electrolyte deficiency is overcorrected or corrected too rapidly. Therefore, early diagnosis and effective treatment of hyponatremia is critical for hyponatremic patients with intracranial disease. ${ }^{30}$

Both SIADH and CSW are potential causes of hyponatremia in patients who have undergone neurosurgery. It may be difficult to distinguish between these two conditions in some cases because their clinical presentations sometimes overlap. The primary distinction lies in the assessment of the patient's volume. The SIADH is characterized by a volume-expanded state, whereas CSW is

Abbreviations used in this paper: $\mathrm{ADH}=$ antidiuretic hormone; $\mathrm{ANP}=$ atrial natriuretic peptide; $\mathrm{BNP}=$ brain natriuretic peptide; $\mathrm{CSW}=$ cerebral salt wasting; $\mathrm{DI}=$ diabetes insipidus; $\mathrm{ECV}=$ effective circulating volume; ICP = intracranial pressure; ICU = intensive care unit; SAH = subarachnoid hemorrhage; SIADH = syndrome of inappropriate secretion of $\mathrm{ADH}$; $\mathrm{TBI}=$ traumatic brain injury. characterized by a volume-contracted state. ${ }^{47}$ It is important to make an accurate diagnosis because treatment differs greatly between these conditions. Fluid restriction is the treatment of choice in SIADH, whereas salt and volume replacement are the treatment for CSW.

Although there are a variety of possible causes of hyponatremia, in this review we will focus on hyponatremia that is commonly encountered in the neurosurgical ICU. Specifically, we will focus on SIADH, the putative associated condition of CSW, and adrenal insufficiency in the settings of TBI, aneurysmal SAH, transsphenoidal surgery for pituitary tumors, and cranial vault reconstruction.

\section{DIFFERENTIAL DIAGNOSIS Hyperosmotic Hyponatremia}

In hyperosmotic (or translocational) hyponatremia disorders, solutes confined to the extracellular compartment induce shifts in transcellular water. ${ }^{1}$ As an example, hyperglycemia or the retention of hypertonic mannitol may result in hyponatremia because water shifts from the intracellular to the extracellular space, causing dehydration of cells. Symptoms common to hyperglycemia include nausea, vomiting, dry axillae and oral mucosae, lower jugular venous pressure, and abdominal pain. ${ }^{68} \mathrm{~A}$ different set of changes is seen when mannitol is given to patients with underlying renal failure or when it is administered in very high doses. ${ }^{10,54}$ Not only does the rise in plasma osmolality cause hyponatremia, but the fluid shift may also lead to volume expansion and possibly pulmonary edema, metabolic acidosis, and hyperkalemia. ${ }^{10}$ 


\section{Isosmotic Hyponatremia}

Isosmotic hyponatremia may be observed in patients who undergo transurethral resection of the prostate or hysterectomy (Fig. 1). During these procedures, patients may absorb large quantities of hyposmotic glycine or sorbitol irrigating solutions leading to a dilutional reduction in the plasma sodium concentration. The extent of isosmotic hyponatremia is related to both the quantity and rate of fluid that is absorbed. ${ }^{34}$ The earliest symptom is nausea, with more severe hyponatremia leading to confusion, disorientation, twitching, seizures, and hypotension. ${ }^{34}$ On the other hand, a less common condition of hyponatremia associated with normal serum osmolality is seen in patients with extreme hyperlipidemia and hyperproteinemia. Also known as "pseudohyponatremia," this finding is based on a laboratory artifact of non-ion-selective techniques to measure serum electrolyte conditions and has been essentially eliminated with the increasing use of ion-selective electrodes for these measurements. ${ }^{1,3,46}$

\section{Hyposmotic Hyponatremia}

Most hyponatremic disorders are associated with hyposmolality. Also known as "dilutional hyponatremia," these disorders may be caused by excessive water intake, but are more commonly caused by water retention. ${ }^{1}$ This subcategory of hyponatremia may be further differentiated according to volume status (Fig. 1). First, volume-expanded hyponatremia occurs when the intake of salt and water exceeds renal and extrarenal losses. The sodium and water retention may be primary, from an increased ECV, or secondary, in response to a decreased ECV. ${ }^{3}$ Diagnoses associated with an increased ECV due to primary water retention by the kidney include acute renal failure and advanced chronic renal failure. Those diagnoses associated with a decreased ECV include congestive heart failure, cirrhosis, nephrotic syndrome, sepsis, anaphylaxis, and pregnancy. ${ }^{1,3}$ The latter conditions are characterized by high plasma concentrations of arginine vasopressin, despite the presence of hypotonicity. The retention of renal

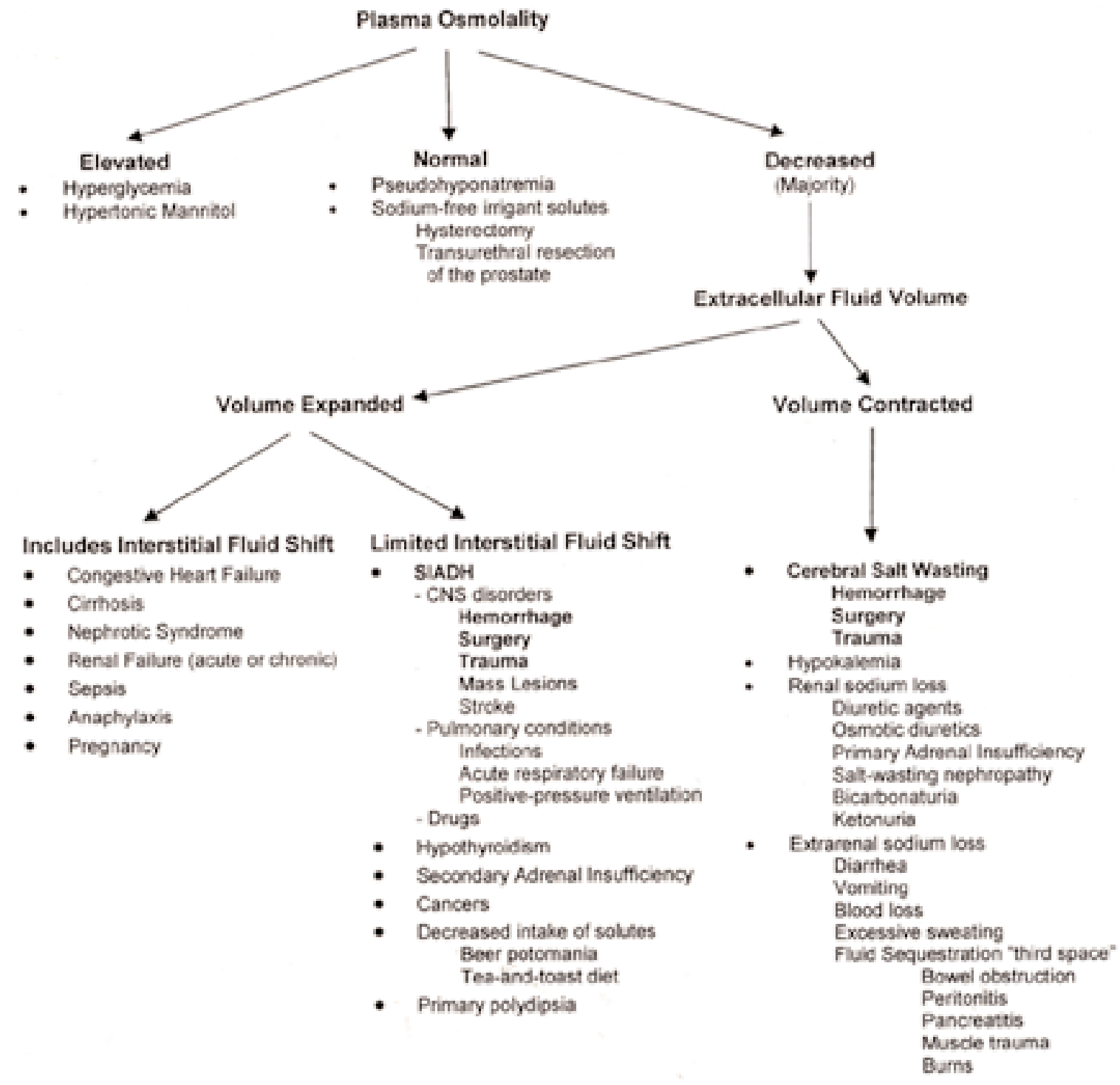

Fig. 1. Flow chart showing the diagnostic approach to hyponatremia. 
sodium and water, which produces hypervolemic hyponatremia and leads to an increased capillary hydrostatic pressure, consequently favors a fluid shift from the intravascular to the interstitial space (Fig. 1). ${ }^{3}$

Other causes of volume-expanded hyponatremia are due to a reduced rate of salt resorption by the diluting segment of the kidney (hypothyroidism, secondary adrenal insufficiency, cancers), sustained nonosmotic release of ADH (SIADH), or a combination of these two factors (Fig. 1). ${ }^{3}$ Less common causes include a decreased intake of solutes and primary polydipsia. In these latter causes of hyponatremia, there is an increase in the intravascular volume with a minimal shift into the interstitial space.

Conversely, volume-contracted hyponatremia is mainly caused by fluid loss from the intravascular space, which is induced by an intrinsic or a secondary renal loss of sodium, an extrarenal loss of sodium, or hypokalemia (Fig. 1). ${ }^{1,3}$ The consequential decrease in tissue perfusion stimulates a release of ADH that is mediated by baroreceptors within the carotid sinus, which sense a reduction in pressure or stretch. The response of baroreceptors to the decrease in volume can overcome the inhibitory effect of serum hyposmolality on ADH secretion-inducing hyponatremia. In conditions of hypokalemia, the depletion of potassium contributes to hyponatremia because the concentration of sodium is determined in part by osmotically active ions present in the total body water, specifically the ratio of exchangeable sodium and potassium. ${ }^{3}$

The complications of hyposmotic hyponatremia occur most often in patients with excessive water retention. ${ }^{1}$ In these conditions, the hypotonic hyponatremia causes transcellular shifts in water from the extracellular into the intracellular space. Because the cranium limits the expansion of the brain by the imposed edema, an increase in ICP develops along with a risk of brain herniation. Fortunately, the degree of swelling is ameliorated as solutes leave the brain tissues within hours, allowing for some water loss within the hypertensive environment. ${ }^{13,45}$ This adaptive process limits the degree of central nervous system complications if the severity of hyponatremia develops slowly. Nevertheless, this adaptation also gives rise to the risk of osmotic demyelination (central pontine myelinolysis), which can develop between 1 and several days after rapid correction of hyponatremia (Fig. 2)..$^{37,43,45,62,69}$ Thus, the clinical manifestations of hyposmotic hyponatremia are more evident when the decrease in the serum sodium concentration is large or when the decrease occurs over a period of hours. ${ }^{7}$ Patients in whom the serum sodium concentration is greater than $125 \mathrm{mmol} / \mathrm{L}$ are usually asymptomatic, whereas those in whom these values are lower may have symptoms that include headache, nausea, vomiting, muscle cramps, lethargy, restlessness, disorienta-

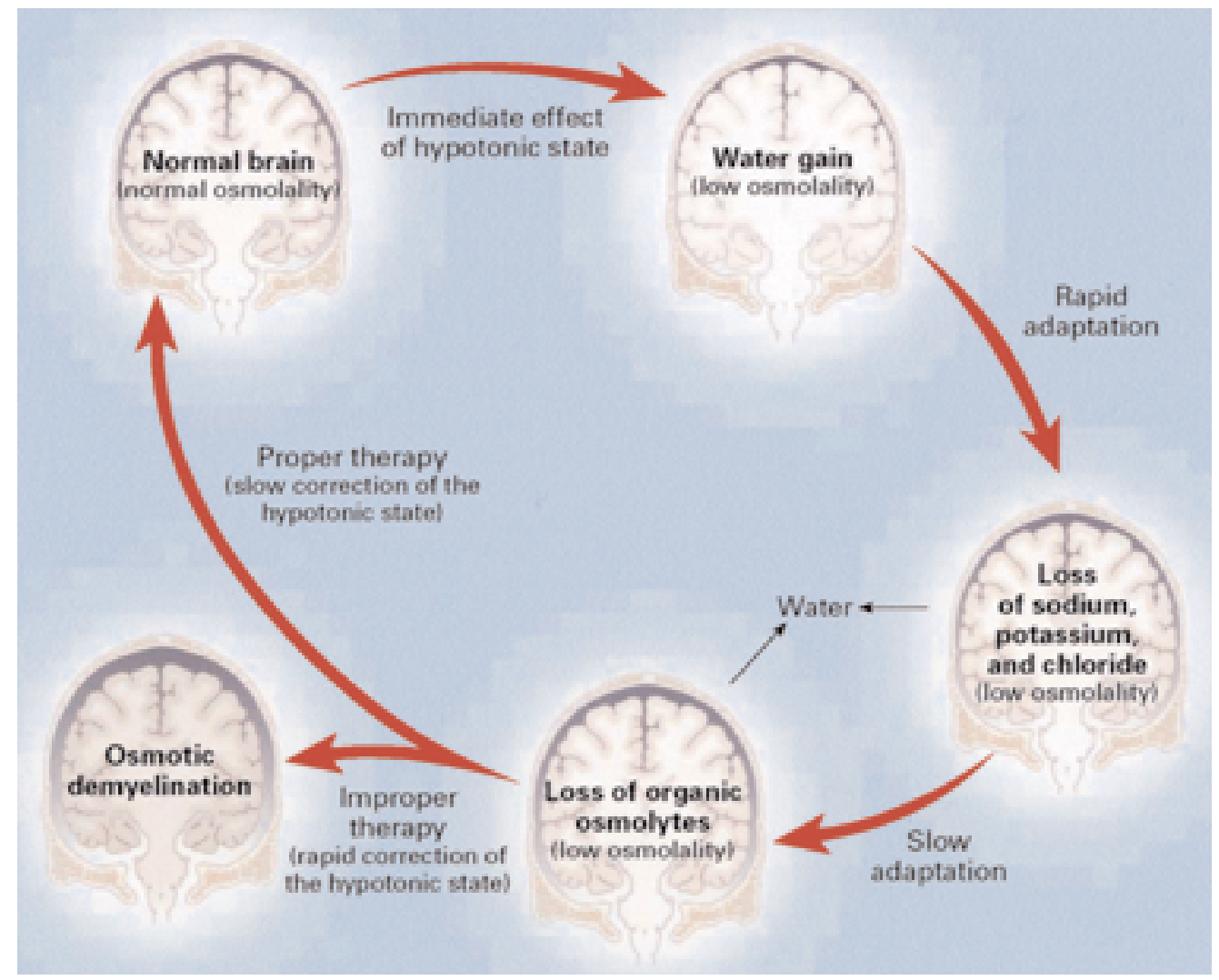

Fig. 2. Effects of hyponatremia on the brain. Minutes after the development of hyponatremia, the decreased osmolality causes swelling of the brain. Rapid adaption occurs within hours as a result of the cellular loss of electrolytes. Slow adaption occurs over several days through the loss of organic osmolytes from brain cells to normalize brain volume. Aggressive correction of hyponatremia may lead to irreversible brain damage (osmotic demyelination); however, proper correction of hyponatremia reestablishes normal osmolality without the risk of brain damage. From Adrogue HJ, Madias NE: Hyponatremia. N Engl J Med 342:1581-1589, 2000; reprinted with permission. 
tion, and depressed reflexes. ${ }^{1,8}$ If, however, the disorder has developed rapidly, complications of severe and rapidly evolving hyponatremia include seizures, coma, permanent brain damage, respiratory arrest, brainstem herniation, and death. ${ }^{1,8}$

\section{Comparison of SIADH and CSW}

Serum osmolality and intravascular fluid volume are the primary stimuli for ADH release. Osmoreceptors in the hypothalamus sense changes in serum osmolality and induce or depress $\mathrm{ADH}$ secretion from the posterior pituitary. Baroreceptors within the carotid sinuses, on the other hand, sense intravascular volume and permit the release of $\mathrm{ADH}$ with volume contraction. In the kidney, $\mathrm{ADH}$ then increases the permeability of the terminal distal tubule and medullary collecting duct to water, leading to the expansion of the ECV. A pronounced elevation of $\mathrm{ADH}$, however, leads to an increase in the ECV, resulting in dilutional hyponatremia.

In SIADH neither hyperosmolality nor volume contraction is the stimulus behind the release of ADH. In cases of SIADH, excessive levels of ADH occur as a result of disease- or drug-induced pituitary release of ADH or the ectopic production of $\mathrm{ADH} .{ }^{30}$ Because of these excessive ADH levels, the diagnosis of SIADH is based on the presence of a low serum level of sodium, an inappropriately concentrated urine compared with serum osmolality, a high urinary sodium level, and the absence of peripheral edema or dehydration with no evidence of adrenal, thyroid, or renal dysfunction. ${ }^{30,53,56}$ Currently, the physiological conditions behind the increased urinary sodium concentrations associated with SIADH is not understood, ${ }^{30}$ although natriuresis associated with SIADH has been attributed to an increase in the glomerular filtration rate and/or a decrease in renal tubular sodium resorption, which is induced by other hormonal or direct neural effects. ${ }^{11,30,41}$ When making the diagnosis of SIADH, it is essential to exclude other causes of hyponatremia that commonly occur in neurological diseases such as edematous states, recent diuretic therapy, and hypovolemic states. ${ }^{30}$ Moreover, the diagnosis of SIADH cannot be made in the presence of severe pain, nausea, stress, or hypotension, because these conditions can stimulate ADH secretion even in the presence of serum hypotonicity. ${ }^{30,51}$

All the changes in electrolyte imbalances observed in SIADH have also been described in CSW; however, the presence of signs of volume depletion (for example, hypotension, decreased skin turgor, or low central venous pressure) with salt wasting distinguishes CSW from SIADH. ${ }^{11,29,30,41,44,51,53,56,70,73,79}$ In essence, the primary distinction between SIADH and CSW lies in the assessment of the ECV. As mentioned earlier, SIADH is an expanded state of ECV due to an ADH-mediated renal water retention, whereas CSW is characterized by a contracted state of ECV due to renal salt wasting. Additional laboratory evidence that relates to the ECV may also help distinguish SIADH from CSW. These include hemoconcentration, albumin concentration, blood urea nitrogen/creatinine ratio, potassium concentration, plasma renin, aldosterone levels, atrial natriuretic factor, plasma urea concentration, and central venous pressure (Table 1). ${ }^{56}$

The mechanism by which intracranial disease leads to CSW is not well understood. Many physicians have pos- tulated that the most probable process involves the disruption of neural input into the kidney and/or the central elaboration of a circulating natriuretic factor. $29,30,44,56,73,79$ Specifically, direct neural effects, an ouabainlike compound, and natriuretic peptides have been implicated in the pathogenesis of CSW. Decreased sympathetic input to the kidney directly and indirectly alters salt and water management and may explain the natriuresis and diuresis seen within CSW. ${ }^{29,56}$ A decrease in sympathetic tone leads to a decreased glomerular filtration rate, a decreased renin release, and a decreased renal tubular sodium resorption. ${ }^{12,21,23,28}$ In addition to a decreased neural input to the kidney, an ouabainlike compound in the brain may play a role in renal salt wasting. Yamada, et al. ${ }^{78}$ found that infusion of digoxin-specific antibodies into the cerebral ventricles blocked the natriuretic response of the rat brain to intracerebroventricular infusion of hypertonic saline. The same investigators also found, however, that intravenous administration of large doses of digoxin-specific antibodies did not block central nervous system-induced natriuresis. ${ }^{78}$ Because of this latter finding and the possible crossreactivity of antiglycoside antibodies with nonglycoside substances, ${ }^{35}$ ouabainlike compound seems less likely to be the sole intermediary of CSW.

Atrial natriuretic peptide and BNP produce several effects that could lead to the clinical syndrome of CSW. Atrial natriuretic peptide is released from the heart in response to atrial stretch and induces vasodilation as well as natriuresis and diuresis..$^{20,72}$ Nevertheless, the findings of several studies contradict the idea that ANP is the primary factor behind CSW. For instance, ANP was found to decrease in the presence of experimental natriuresis induced by intracerebroventricular administration of hypertonic saline, ${ }^{48}$ ANP was not elevated after $\mathrm{SAH},{ }^{25,36}$ and normal levels of ANP were found in patients with CSW associated with intracerebral hemorrhage ${ }^{73}$ and surgery for pituitary adenomas. ${ }^{79}$

Brain natriuretic peptide is secreted by the cardiac ventricles in response to increased pressure or stretch, and displays biological effects similar to those of ANP; ${ }^{30}$ furthermore, this substance has also been localized to the hypothalamus. ${ }^{64}$ Berendes, et al., ${ }^{12}$ demonstrated that concentrations of BNP significantly correlated with both urinary sodium excretion and ICP in patients with SAH both

TABLE 1

Comparison of laboratory values and treatment in the differential diagnosis of SIADH or CSW

\begin{tabular}{lcc}
\hline \hline Laboratory Value or Treatment & SIADH & CSW \\
\hline ECV & $\begin{array}{c}\text { increased or } \\
\text { no change } \\
\text { increased } \\
\text { central venous pressure }\end{array}$ & decreased \\
blood urea nitrogen/ & decreased \\
creatinine ratio & no change & increased \\
serum albumin concentration & normal & increased \\
serum potassium concentration & decreased or & increased or \\
& no change & no change \\
serum uric acid concentration & decreased & decreased or \\
no change & normal & increased \\
hematocrit & fluid restriction & salt \& fluid \\
treatment & & replacement \\
& &
\end{tabular}


before surgery and through postoperative Day 8. Intracranial disease may stimulate the sympathetic release of cardiac BNP, which may suppress aldosterone secretion and alter renal function. ${ }^{12,66}$ In addition, BNP may be directly released by the brain when this organ is damaged, thereby limiting extreme rises in ICP. ${ }^{12}$

Making an accurate diagnosis between SIADH and CSW in patients undergoing neurosurgery who develop hyponatremia is important because the treatment of each condition is quite different. ${ }^{56}$ Fluid restriction is the treatment of choice in patients with SIADH because the primary abnormality is ECV expansion, whereas vigorous salt replacement is required in patients with CSW because of ECV contraction (Fig. 3). ${ }^{24,55}$ Failure to distinguish properly between these disorders can potentially result in adverse outcomes. Specifically, there is the potential for an administration of intravenous saline in patients with SIADH to result in symptomatic hyponatremia, because renal handling of water is impaired but renal management of sodium is not. Just as fluid administration may potentially worsen the underlying neurological condition in the setting of SIADH, fluid restriction may exacerbate the underlying neurological condition in the setting of CSW. ${ }^{76} \mathrm{In}$ a retrospective study of patients with $\mathrm{SAH}$, Wijdicks, et al. ${ }^{76}$ found that in 21 of 26 patients treated for hyponatremia with fluid restriction cerebral infarction developed. Although the volume status in these patients was not defined, the authors raised the possibility that CSW was the cause of hyponatremia in many of these patients..$^{55}$ Consequently, patients with CSW require volume replenishment with intravenous saline or administration of salt tablets instead of fluid restriction until the transient (3-4 weeks) condition of renal salt wasting resolves (Fig. 3). ${ }^{24,55}$

\section{Traumatic Brain Injury}

Hyponatremia is frequently seen in patients suffering from TBI. ${ }^{26,71,80}$ Arieff, et al., ${ }^{6}$ found that following TBI, the acute onset of severe hyponatremia was associated with either poor neurological outcome or death in those patients with a sudden onset of seizures, followed by coma, apnea, and brainstem compression. Horn and Glenn ${ }^{32}$ screened patients with severe head injury and found that $20 \%$ suffered from one or more hormone disturbances. The most frequent endocrine disturbance as well as the most common cause of hyponatremia in these patients was SIADH. Although less common, hyponatremia also occurred in the presence of extracellular fluid depletion in the setting of renal sodium loss in an excess of water, that is, CSW. ${ }^{32,61}$ As described earlier, the most important differential diagnosis of acute hyponatremia includes SIADH, CSW, and acute adrenal insufficiency. Unfortunately, the clinical picture and the laboratory data can be initially misleading because the diagnostic criteria of SIADH, CSW, and adrenal insufficiency may overlap.

After several days in the ICU, patients with a recent TBI often excrete very large quantities of sodium chloride. ${ }^{30}$ Urine examination often reveals a negative balance for sodium on one of those initial days in the ICU, typically demonstrating high urinary concentrations of sodium, potassium, and chloride in the setting of hyponatremia. ${ }^{15}$ These findings usually provide the basis on which the clinical diagnosis of CSW may be made. ${ }^{15,30}$ Nevertheless, a stimulus to excrete sodium and chloride ions, such as expanded extracellular fluid volume, must be ruled out. It is difficult to ascertain whether extracellular fluid, or even more importantly, the ECV is contracted on clinical grounds unless changes are quite marked. ${ }^{16}$ Carolotti, et

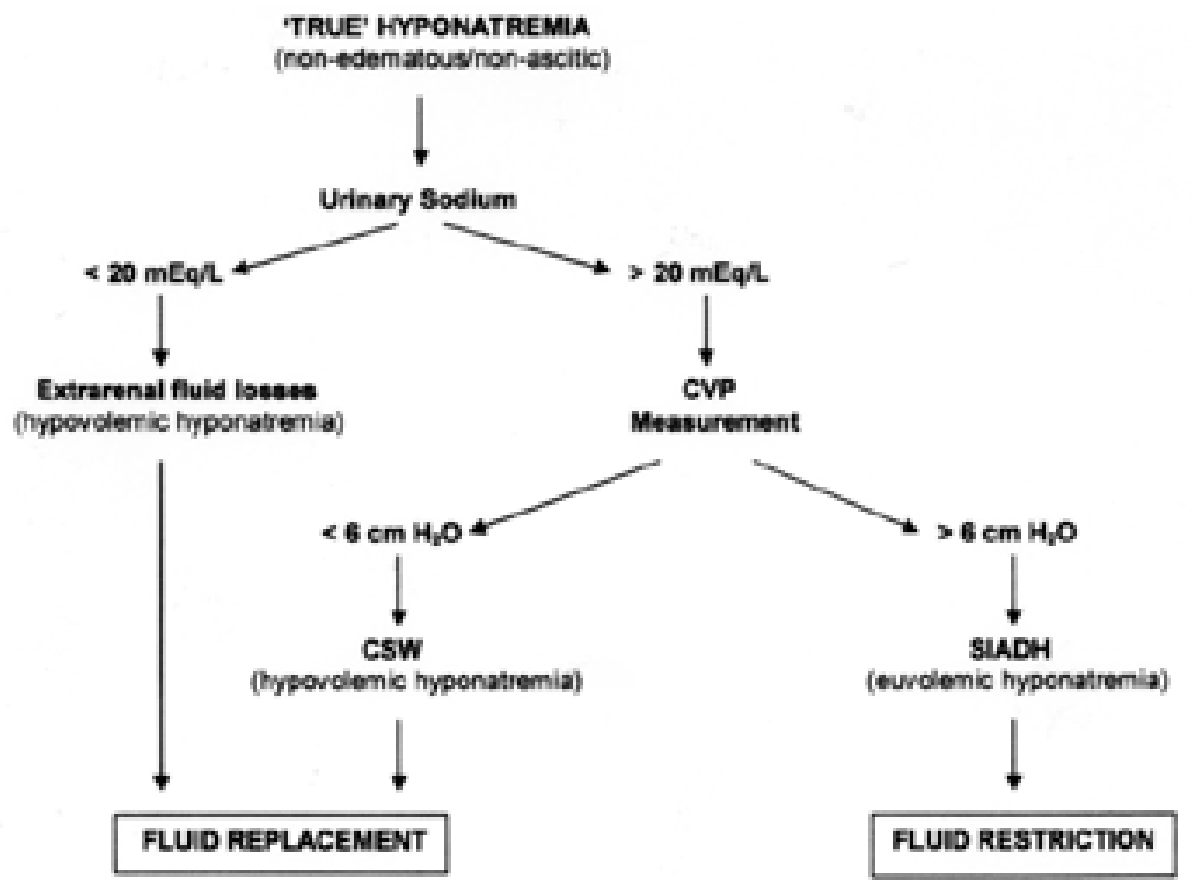

Fig. 3. Algorithm for the evaluation and treatment of patients who have undergone neurosurgery and have hyponatremia and natriuresis. CVP = central venous pressure. From Docci D, Cremonini AM, Nasi MT, et al: Hyponatremia with natiuresis in neurosurgical patients. Nephro Dial Transplant 15:1707-1708, 2000; by permission of Oxford University Press. 
al. ${ }^{15}$ have proposed the use of common electrolyte measurements to differentiate between SIADH and CSW. These investigators determined the total body fluid based on the height and weight of the patient and then multiplied this by either the sum of the serum sodium and potassium concentrations or the serum chloride concentration. ${ }^{15,60}$ Because CSW is dependent on ongoing natriuresis, despite ECV contraction, measurement of sodium and potassium ions or chloride ions along with total body water allows an accurate estimation of the mass balance over time. ${ }^{15}$ These data then provide information to support or refute a clinical diagnosis of CSW. ${ }^{15}$

Another complicating factor is the occurrence of an acute adrenal crisis within the population of head-injured patients, which may result from bilateral adrenal hemorrhage. ${ }^{18}$ In a study conducted by Atchinson and colleagues, ${ }^{9}$ in a group of patients with TBI absolute hyponatremia but no adrenal insufficiency was associated with cognitive impairments that were very similar to those symptoms frequently seen in primary adrenal insufficiency. ${ }^{71}$ The development of acute adrenal crisis after adrenal infarction or hemorrhage may lead to fulminant shock and death, if not recognized and acted on quickly. ${ }^{71}$ A decrease in cortisol levels acts as a stimulus for ADH secretion. ${ }^{14}$ Although SIADH and primary adrenal insufficiency may present with similar values of osmolality and urine electrolyte levels, other causes of hyponatremia should be considered when the patient remains unresponsive to standard treatments for SIADH. ${ }^{71}$ Another key to the recognition of adrenal insufficiency is the finding of relative hyperkalemia in the context of hyponatremia. ${ }^{71}$ This is a classic depiction of electrolytes seen with a deficiency of mineralocorticoid activity, which leads to urinary sodium losses, impaired excretion of potassium, and hydrogen ion retention. ${ }^{71}$ Additionally, azotemia with mild elevations in both blood urea nitrogen and creatinine commonly occurs with primary adrenal insufficiency. ${ }^{71}$

\section{Aneurysmal SAH}

Abnormal serum levels of sodium are frequently observed during the acute period (4-10 days) ${ }^{40,48,49,57,59,75}$ following aneurysmal SAH. ${ }^{57}$ According to several reports, 29 to $43 \%$ of patients with SAH experience hyponatremia. ${ }^{31,40,57,75,76}$ When $\mathrm{SAH}$ is stratified according to the site of rupture, Sayama, et al., ${ }^{59}$ found that patients with ruptured anterior communicating aneurysms had a significantly higher incidence of hyponatremia (51\%) than patients with ruptured middle cerebral artery aneurysms $(18 \%)$. The presence of hyponatremia is significant, because it is recognized as a risk factor for, as well as a predictor of, vasospasm and cerebral ischemia. ${ }^{49,59,75}$ Specifically, Morinaga, et al., ${ }^{49}$ found that $84 \%$ of patients with hyponatremia demonstrated symptomatic cerebral vasospasm. Cerebral ischemia also occurs more frequently in patients with hyponatremia than in those without hyponatremia, although Hasan, et al. ${ }^{31}$ did not find any difference in mortality rates. Qureshi and coworkers ${ }^{57}$ also did not note a difference in outcomes in patients who had hyponatremia and hypothesized that aggressive fluid management and correction of hyponatremia may have countered the negative effects. ${ }^{8}$

Hyponatremia related to $\mathrm{SAH}$ is thought to occur sec- ondary to the release of BNP, ${ }^{12,34,40,63,66}$ but the stimulus for its release is not clear. ${ }^{63}$ It was first suspected that ANP was responsible for the hyponatremia, but ANP concentrations did not correlate with hyponatremia, indicating the presence of another factor. ${ }^{63}$ Subsequently, BNP concentrations have been shown to be elevated immediately after SAH, compared with levels in control patients. Moreover, BNP concentrations have been demonstrated to increase further in patients in whom cerebral vasospasm developed. Svri and colleagues ${ }^{63}$ found that BNP levels increased progressively in the 1 st week after SAH, peaking on Days 7 through 9, and that these levels correlated well with the severity of SAH as well as with the severity of the vasospasm.

As mentioned earlier, hyponatremia after SAH is associated with an aggressive natriuresis that places the patient at risk for ischemic complications due to volume depletion. Additionally, the low serum concentrations of sodium accentuate cerebral edema. The standard of care is aggressive hypervolemic therapy for intravascular volume expansion and sodium supplementation. It is noteworthy that water replacement causes hyponatremia, and increased sodium intake does not prevent a negative sodium balance, but actually enhances urinary sodium excretion, natriuresis, and osmotic diuresis. ${ }^{48,50}$ Aggressive hypervolemic therapies are not always effective on their own and some authors advocate the use of fludrocortisone or hydrocortisone, which inhibit natriuresis. ${ }^{48,50}$ These agents function by promoting sodium resorption in the kidney through their mineralocorticoid effects. ${ }^{7}$ Mori and coworkers $^{48}$ found that fludrocortisone reduced the need for sodium replacement and water intake and decreased urine output and excretion of sodium. In a study by Moro, et al. ${ }^{50}$ hydrocortisone therapy resulted in lower urine volumes, lower infusion volumes required for hypervolemic therapy, and higher central venous pressures. Hyponatremia did not develop in any patient who received fludrocortisone or hydrocortisone. ${ }^{48,50}$ In these studies, no adverse effects were caused by the steroids. ${ }^{48,50}$

\section{Transsphenoidal Surgery for Pituitary Tumors}

Diabetes insipidus is a deficiency of ADH that may result from the dysfunction of neurons that originate in the supraoptic and paraventricular nuclei of the hypothalamus; it commonly occurs following transsphenoidal surgery for pituitary tumors (Fig. 4). Postoperatively, an ADH deficiency normally leads to polyuria (urine output $>5 \mathrm{ml} / \mathrm{kg} / \mathrm{hr}$ ) with an excessive free water loss that generates a urine/plasma osmolality ratio lower than $1.5 .^{2}$ Therapeutic intervention includes maintaining an appropriate plasma osmolality via adequate fluid replacement along with administration of desmopressin to avoid hypernatremic hypovolemia.

Diabetes insipidus may coexist with CSW, however. ${ }^{2}$ Cerebral salt wasting is thought to occur secondarily to the release of BNP after transsphenoidal resection of pituitary adenomas $^{4,23}$ and CSW is possibly the cause of hyponatremia in cases in which there are normal levels of ADH. As mentioned earlier, the release of BNP may be due to a sympathetic stimulation, although the exact association between BNP and hyponatremia in patients after transsphenoidal resection of pituitary adenomas remains to be 


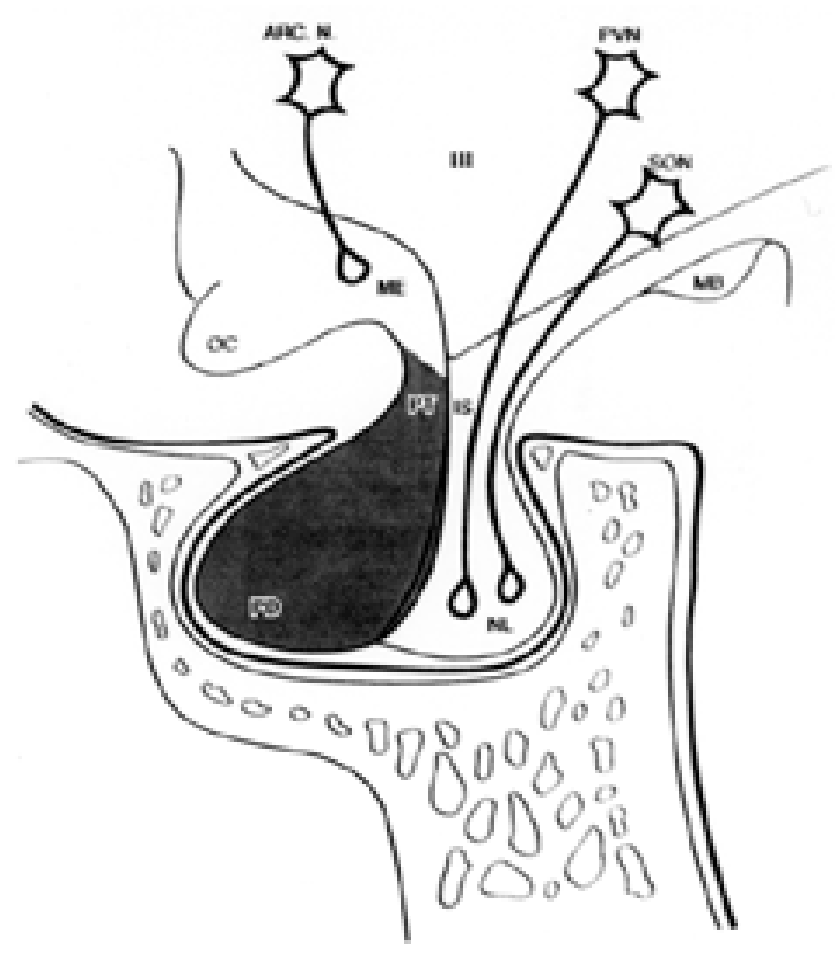

Fig. 4. A sagittal diagram of the anatomy of the pituitary gland, hypothalamus, and pituitary stalk. The anterior gland, or adenohypophysis, is composed of the pars distalis (PD), the rudimentary pars intermedia, and the pars tuberalis (PT). The posterior gland, or neurohypophysis (located in the hypothalamus), is composed of the median eminence (ME), the infundibular stem (IS), and the neural lobe (NL). The large neurons located in the supraoptic nucleus (SON) and the paraventricular nucleus (PVN) project to the neural lobe, where they store and release oxytocin and vasopressin into the systemic circulation under the appropriate stimulus. ARC. $\mathrm{N} .=$ arcuate nucleus; $\mathrm{MB}=$ mammillary bodies; $\mathrm{OC}=$ optic chiasm. Figure 35-3 from Couldwell WT, Simard MF, Weiss MH, et al: Pituitary and Adrenal, in Schwartz SI (ed): Principles of Surgery, ed 7, vol 2, New York: McGraw-Hill, pp 1613-1659; reprinted with permission.

elucidated. In any case, patients with concurrent DI and CSW may have an increased urine output that has been incorrectly attributed to poorly controlled DI rather than natriuresis. ${ }^{2}$ If the syndromes are concomitant, an increase in the desmopressin dose would be inappropriate because it would lead to an increase in renal free water resorption and further exacerbate the hyponatremia. ${ }^{2}$ In these uncommon cases, therapeutic intervention should include sodium and fluid replacement along with the administration of desmopressin with close monitoring of plasma electrolytes and osmolality.

Following pituitary surgery DI has also been found to occur as a triple response. ${ }^{2}$ This is characterized by an initial 4- to 8-day period of DI followed by a transient remission or an excessive release of ADH (similar to SIADH) lasting 1 to 14 days, which concludes with the recurrence of often permanent DI. ${ }^{2}$

Conversely, symptomatic delayed hyponatremia has been reported in patients who have undergone transsphenoidal surgery for pituitary adenomas $(2.3-16 \%) .38,65,74$ The time course of delayed hyponatremia after transsphe- noidal pituitary removal is quite characteristic. ${ }^{38}$ Patients usually become hyponatremic approximately 5 days following surgery with the nadir and symptoms occurring a mean of 7 to 8 days postoperatively. ${ }^{19,38,52,65,74}$ The common clinical manifestations are nausea, vomiting, headache, malaise, and dizziness. The lack of clinical predictors for the development of this delayed hyponatremia, other than a large tumor size (macroadenomas), suggests that tumor removal is responsible for a transient hypothalamopituitary disturbance..$^{38}$ The release of ADH stores from those surgically manipulated neurohypophysial cells is the likely mechanism resulting in an SIADH-like syndrome with fluid retention and natriuresis. ${ }^{38}$ Elevated serum levels of $\mathrm{ADH}$ have been demonstrated in patients in whom hyponatremia develops following transsphenoidal surgery for pituitary adenomas $;^{19,67}$ however, this is not always the case. ${ }^{63}$

Many studies support the concept that delayed hyponatremia following transsphenoidal surgery is based on an adrenocortical insufficiency. ${ }^{22,39}$ Secondary adrenal insufficiency may indeed be the causative factor behind the SIADH-like syndrome because cortisol has been shown to be an inhibitor of vasopressin secretion. ${ }^{27,58}$ Diederich, et al.,22 found that ADH was elevated in a majority of patients with severe euvolemic hyponatremia. These same investigators went on to treat seven patients with hydrocortisone and found a subsequent decrease in ADH levels. Such findings need to be considered in the context of an acute illness in which basal cortisol levels may be subnormal. ${ }^{22}$ Even so, a high clinical suspicion should be maintained in treating severely hyponatremic patients for the possibility of a cortisol-deficient SIADH-like syndrome. Low doses of hydrocortisone in a hyponatremic patient without hypopituitarism will seldom be harmful, whereas the failure to give hydrocortisone to a patient with the disorder may have deleterious consequences. ${ }^{22}$ In differentiating between the triple response, as described earlier, and delayed hyponatremia, Kelly, et al., ${ }^{38}$ found that 14 of 99 patients who underwent transsphenoidal resection of a pituitary adenoma experienced transient DI requiring treatment with desmopressin acetate. In none of these cases was DI permanent and no patient in whom DI developed experienced delayed hyponatremia.

Given the possibility of DI, CSW, SIADH-like syndrome, or secondary adrenal insufficiency following transsphenoidal resection of pituitary adenomas, plasma osmolality, urine osmolality, and ECV status must be determined to provide appropriate treatment. Because the onset of delayed hyponatremia usually occurs after the patient has been discharged from the hospital, the patient should be educated and forewarned. If this individual experiences headache, nausea, vomiting, or fatigue, a serum level of sodium should be obtained. If hyponatremia is confirmed, treatment with fluid restriction and salt replacement should be instituted.

\section{Cranial Vault Reconstruction for Craniosynostosis}

Hyponatremia frequently occurs after cranial vault reconstruction for craniosynostosis in pediatric patients. $33,42,77$ The exact mechanism for postoperative hyponatremia after cranial vault reconstruction remains controversial. Initial reports have indicated that the cause 
is SIADH. Hudgins, et al. ${ }^{33}$ reported two cases in which the patients experienced symptomatic hyponatremia after cranial vault remodeling for late sagittal synostosis. One patient became obtunded and one had generalized seizures. Interestingly, both of these patients lost at least $1 \mathrm{~L}$ of blood during the operation. These authors attributed the hyponatremia to SIADH precipitated by large fluid shifts during surgery.

Others have opposed this theory and have favored CSW as the primary cause of postoperative hyponatremia in these patients. ${ }^{28,42}$ Ganong and Kappy ${ }^{28}$ described CSW, as opposed to SIADH, in two children with central nervous system insults. The two patients were successfully treated with fluid and sodium replacement. Levine, et al., ${ }^{42}$ also analyzed their series of children who underwent craniofacial reconstruction and found a positive correlation between cranial vault remodeling and CSW. All their patients who had undergone uncomplicated cranial vault remodeling procedures experienced transient hyponatremia $(128-133 \mathrm{mEq} / \mathrm{L})$, despite receiving postoperative normal saline resuscitation. Most of the patients exhibited increased urine output, normal or increased urine levels of sodium, and low serum levels of sodium, suggesting the diagnosis of CSW.

The clinician should be aware and recognize changes in fluids and electrolytes in patients who have undergone cranial vault reconstruction. Daily monitoring of serum levels of sodium and maintenance of normonatremia is important to prevent deleterious effects. Levine, et al., ${ }^{42}$ have suggested that administration of hypotonic solution or fluid restriction may be the incorrect treatment and may exacerbate hyponatremia. In our experience with pediatric patients who undergo craniofacial reconstruction surgery, postoperative hyponatremia is usually transient and asymptomatic. We routinely administer normal saline solution and monitor daily serum levels of sodium. If the serum sodium level decreases below 126 to $127 \mathrm{mEq} / \mathrm{L}$, we administer hypertonic saline.

\section{CONCLUSIONS}

Hyponatremia, caused by either SIADH or CSW, is commonly observed in many patients with intracranial disease who undergo neurosurgery, especially in the setting of TBI and aneurysmal SAH. It is of paramount importance that the physician differentiate between SIADH and CSW because of the disparate nature of the treatments of these two entities. Improper treatment can worsen the underlying condition and may result in poor neurological outcomes. The primary distinction between SIADH and CSW rests on the assessment of the ECV of the patient.

\section{References}

1. Adrogue HJ, Madias NE: Hyponatremia. N Engl J Med 342: 1581-1589, 2000

2. Albanese A, Hindmarsh P, Stanhope R: Management of hyponatraemia in patients with acute cerebral insults. Arch Dis Child 85:246-251, 2001

3. Andreoli TE, Abul-Ezz SR: Fluid and electrolyte disorders, in Andreoli TE, Carpenter CCJ, Griggs RC, et al (eds): Cecil Essentials of Medicine, ed 5. Philadelphia: WB Saunders, 2001, pp 238-252

4. Andrews BT, Fitzgerald PA, Tyrell JB, et al: Cerebral salt wast- ing after pituitary exploration and biopsy: case report. Neurosurgery 18:469-471, 1986

5. Arieff AI: Hyponatremia, convulsions, respiratory arrest, and permanent brain damage after elective surgery in healthy women. N Engl J Med 314:1529-1535, 1986

6. Arieff AI, Ayus JC, Fraser CL: Hyponatraemia and death or permanent brain damage in healthy children. BMJ 304: 1218-1222, 1992

7. Arieff AI, Guisado R: Effects on the central nervous system of hypernatremic and hyponatremic states. Kidney Int 10: 104-116, 1976

8. Arieff AI, Llach F, Massry SG: Neurological manifestations and morbidity of hyponatremia: correlation with brain water and electrolytes. Medicine 55:121-129, 1976

9. Atchinson JW, Wacherndorf J, Haddock D, et al: Hyponatremia-associated cognitive impairment in traumatic brain injury. Brain Inj 7:347-352, 1993

10. Aviram A, Pfau A, Czaczkes JW, et al: Hyperosmolality with hyponatremia, caused by inappropriate administration of mannitol. Am J Med 42:648-650, 1967

11. Bartter FC, Schwartz WB: The syndrome of inappropriate secretion of antidiuretic hormone. Am J Med 42:790-806, 1967

12. Berendes E, Walter M, Cullen P, et al: Secretion of brain natriuretic peptide in patients with aneurysmal subarachnoid haemorrhage. Lancet 349:245-249, 1997

13. Berl T: Treating hyponatremia: damned if we do and damned if we don't. Kidney Int 37:1006-1018, 1990

14. Burke CW: Adrenocortical insufficiency. Clin Endocrinol Metab 14:947-976, 1985

15. Carlotti APCP, Bohn D, Rutka JT, et al: A method to estimate urinary electrolyte excretion in patients at risk for developing cerebral salt wasting. J Neurosurg 95:420-424, 2001

16. Chung HM, Kluge R, Schrier RW, et al: Clinical assessment of extracellular fluid volume in hyponatremia. Am J Med 83: 905-908, 1987

17. Chung HM, Kluge R, Schrier RW, et al: Postoperative hyponatremia. A prospective study. Arch Intern Med 146:333-336, 1986

18. Claussen MS, Landercasper J, Cogbill TH: Acute adrenal insufficiency presenting as shock after trauma and surgery: three cases and review of the literature. J Trauma 32:94-100, 1992

19. Cusick JF, Hagen TC, Findling JW: Inappropriate secretion of antidiuretic hormone after transsphenoidal surgery for pituitary tumors. N Engl J Med 311:36-38, 1984

20. de Zeeuw D, Janssen WM, de Jong PE: Atrial natriuretic factor: its (patho)physiological significance in humans. Kidney Int 41:1115-1133, 1992

21. DiBona GF: Neural control of renal function in health and disease. Clin Auton Res 4:69-74, 1994

22. Diederich S, Franzen N, Bahr V, et al: Severe hyponatremia due to hypopituitarism with adrenal insufficiency: report on 28 cases. Eur J Endocrinol 148:609-617, 2003

23. Diringer M, Ladenson PW, Borel C, et al: Sodium and water regulation in a patient with cerebral salt wasting. Arch Neurol 46:928-930, 1989

24. Docci D, Cremonini AM, Nasi MT, et al: Hyponatraemia with natriuresis in neurosurgical patients. Nephrol Dial Transplant 15:1707-1708, 2000

25. Doczi T, Joo F, Vecsernyes M, et al: Increased concentration of atrial natriuretic factor in the cerebrospinal fluid of patients with aneurysmal subarachnoid hemorrhage and raised intracranial pressure. Neurosurgery 23:16-19, 1988

26. Donati-Genet PC, Dubuis JM, Girardin E, et al: Acute symptomatic hyponatremia and cerebral salt wasting after head injury: an important clinical entity. J Pediatr Surg 36: 1094-1097, 2001

27. Erkut ZA, Pool C, Swaab DF: Glucocorticoids suppress corticotropin-releasing hormone and vasopressin expression in human hypothalamic neurons. J Clin Endocrinol Metab 83: 2066-2073, 1998 
28. Ganong CA, Kappy MS: Cerebral salt wasting in children. The need for recognition and treatment. Am J Dis Child 147: 167-169, 1993

29. Harrigan MR: Cerebral salt wasting syndrome. Crit Care Clin 17:125-138, 2001

30. Harrigan MR: Cerebral salt wasting syndrome: a review. Neurosurgery 38:152-160, 1996

31. Hasan D, Wijdicks EF, Vermeulen M: Hyponatremia is associated with cerebral ischemia in patients with aneurysmal subarachnoid hemorrhage. Ann Neurol 27:106-180, 1990

32. Horn LJ, Glenn MB: Update in pharmacology: pharmacology interventions in neuroendocrine disorders following traumatic brain injury, part two. J Head Trauma Rehabil 3:86-89, 1988

33. Hudgins RJ, Burstein FD, Boydston WR: Total calvarial reconstruction for sagittal synostosis in older infants and children. J Neurosurg 78:199-204, 1993

34. Isotani E, Suzuki R, Tomita K, et al: Alterations in plasma concentrations of natriuretic peptides and antidiuretic hormone after subarachnoid hemorrhage. Stroke 25:2198-2203, 1994

35. Jackowski A: Disordered sodium and water in neurosurgery. $\mathbf{B r}$ J Neurosurg 6:173-176, 1992

36. Juul R, Edvinsson L, Ekman R, et al: Atrial natriuretic peptideLI following subarachnoid haemorrhage in man. Acta Neurochir 106:18-23, 1990

37. Karp BI, Laureno R: Pontine and extrapontine myelinolysis: a neurologic disorder following rapid correction of hyponatremia. Medicine 72:359-373, 1993

38. Kelly DF, Laws ER Jr, Fossett D: Delayed hyponatremia after transsphenoidal surgery for pituitary adenoma. Report of nine cases. J Neurosurg 83:363-367, 1995

39. Kroll M, Juhler M, Lindholm J: Hyponatraemia in acute brain disease. J Intern Med 232:291-297, 1992

40. Kurokawa Y, Uede T, Ishiguro M, et al: Pathogenesis of hyponatremia following subarachnoid hemorrhage due to ruptured cerebral aneurysm. Surg Neurol 46:500-508, 1996

41. Lester MC, Nelson PB: Neurological aspects of vasopressin release and the syndrome of inappropriate secretion of antidiuretic hormone. Neurosurgery 8:735-740, 1981

42. Levine JP, Stelnicki E, Weiner HL, et al: Hyponatremia in the postoperative craniofacial pediatric patient population: a connection to cerebral salt wasting syndrome and management of the disorder. Plast Reconstr Surg 108:1501-1508, 2001

43. Lien YH, Shapiro JI, Chan L: Study of brain electrolytes and organic osmolytes during correction of chronic hyponatremia. Implications for the pathogenesis of central pontine myelinolysis. J Clin Invest 88:303-309, 1991

44. Lolin Y, Jackowski A: Hyponatraemia in neurosurgical patients: diagnosis using derived parameters of sodium and water homeostasis. Br J Neurosurg 6:457-466, 1992

45. Luareno R, Karp BI: Myelinolysis after correction of hyponatremia. Ann Intern Med 126:57-62, 1997

46. Maas AHJ, Siggaard-Andersen O, Weisberg HF, et al: Ion-selective electrodes for sodium and potassium: a new problem of what is measured and what should be reported. Clin Chem 31: 482-485, 1985

47. Maesaka JK, Gupta S, Fishbane S: Cerebral salt-wasting syndrome: does it exist? Nephron 82:100-109, 1999

48. Mori T, Kaytayama Y, Kawamata T, et al: Improved efficiency of hypervolemic therapy with inhibition of natriuresis by fludrocortisone in patients with anuerysmal subarachnoid hemorrhage. J Neurosurg 91:947-952, 1999

49. Morinaga K, Hayashi S, Matsumoto Y, et al: [Hyponatremia and cerebral vasospasm in patients with aneurysmal subarachnoid hemorrhage.] No To Shinkei 44:629-632, 1992 (Jpn)

50. Moro N, Katayama Y, Kojima J, et al: Prophylactic management of excessive natriuresis with hydrocortisone for efficient hypervolemic therapy after subarachnoid hemorrhage. Stroke 34:2807-2811, 2003

51. Moses AM, Streeten DHP: Disorders of the neurohypophysis, in Isselbacher KJ, Braunwald E, Wilson JD, et al (eds): Harrison's Principles of Internal Medicine, ed 13. New York: McGraw-Hill, 1994, pp 1921-1930

52. Murty GE, Lamballe P, Welch AR: Early inappropriate secretion of antidiuretic hormone after trans-sphenoidal pituitary adenomectomy. J Laryngol Otol 104:894-895, 1990

53. Nelson PB, Seif SM, Maroon JC, et al: Hyponatremia in intracranial disease: perhaps not the syndrome of inappropriate secretion of antidiuretic hormone (SIADH). J Neurosurg 55: 938-941, 1981

54. Oster JR, Singer I: Hyponatremia, hyposmolality, and hypotonicity: tables and fables. Arch Intern Med 159:333-336, 1999

55. Pamler BF: Hyponatraemia in a neurosurgical patient: syndrome of inappropriate antidiuretic hormone secretion versus cerebral salt wasting. Nephrol Dial Transplant 15:262-268, 2000

56. Palmer BF: Hyponatremia in patients with central nervous system disease: SIADH versus CSW. Trends Endocrinol Metab 14:182-187, 2003

57. Qureshi AI, Suri MFK, Sung GY, et al: Prognostic significance of hypernatremia and hyponatremia among patients with aneurysmal subarachnoid hemorrhage. Neurosurgery 50: 749-756, 2002

58. Raff H: Glucocorticoid inhibition of neurohypophysial vasopressin secretion. Am J Physiol 252:R635-R644, 1987

59. Sayama T, Inamura T, Matsushima T, et al: High incidence of hyponatremia in patients with ruptured anterior communicating artery aneurysms. Neurol Res 22:151-155, 2000

60. Spital A, Sterns RD: The paradox of sodium's volume of distribution. Why an extracellar solute appears to distribute over total body water. Arch Intern Med 149:1255-1257, 1989

61. Steinbok P, Thompson GB: Metabolic disturbances after head injury: abnormalities of sodium and water balance with special reference to the effects of alcohol intoxication. Neurosurgery 3:9-15, 1978

62. Sterns RH, Riggs JE, Schochet SS Jr: Osmotic demyelination syndrome following correction of hyponatremia. N Engl J Med 314:1535-1542, 1986

63. Sviri GE, Shik V, Raz B, et al: Role of brain natriuretic peptide in cerebral vasospasm. Acta Neurochir 145:851-860, 2003

64. Takahashi K, Totsune K, Sone M, et al: Human brain natriuretic peptide-like immunoreactivity in human brain. Peptides 13: 121-123, 1992

65. Talyor SL, Tyrrell JB, Wilson CB: Delayed onset of hyponatremia after transsphenoidal surgery for pituitary adenomas. Neurosurgery 37:649-654, 1995

66. Tomida M, Muraki M, Uemura K, et al: Plasma concentrations of brain natriuretic peptide in patients with subarachnoid hemorrhage. Stroke 29:1584-1587, 1998

67. Ultmann MC, Hoffman GE, Nelson PB, et al: Transient hyponatremia after damage to the neurohypophyseal tracts. Neuroendocrinology 56:803-811, 1992

68. Umpierrez G, Freire AX: Abdominal pain in patients with hyperglycemic crises. J Crit Care 17:63-67, 2002

69. Videen JS, Michaelis T, Pinto P, et al: Human cerebral osmolytes during chronic hyponatremia. A proton magnetic resonance spectroscopy study. J Clin Invest 95:788-793, 1995

70. Vingerhoets F, de Tribolet N: Hyponatremia hypo-osmolarity in neurosurgical patients. "Appropriate secretion of $\mathrm{ADH}$ " and "cerebral salt wasting syndrome". Acta Neurochir 91:50-54, 1988

71. Webster JB, Bell KR: Primary adrenal insufficiency following traumatic brain injury: a case report and review of the literature. Arch Phys Med Rehabil 78:314-318, 1997

72. Weidmann P, Hasler L, Gnadinger MP, et al: Blood levels and renal effects of atrial natriuretic peptide in normal man. J Clin Invest 77:734-742, 1986

73. Weinand ME, O'Boynick PL, Goetz KL: A study of serum antidiuretic hormone and atrial natriuretic peptide levels in a series 
of patients with intracranial disease of hyponatremia. Neurosurgery 25:781-785, 1989

74. Whitaker SJ, Meanock CI, Turner GF, et al: Fluid balance and secretion of antidiuretic hormone following transsphenoidal surgery. A preliminary series. J Neurosurg 63:1985, 1985

75. Wijdicks EF, Schievink WI, Burnett JC Jr: Natriuretic peptide system and endothelin in aneurysmal subarachnoid hemorrhage. J Neurosurg 87:275-280, 1997

76. Wijdicks EF, Vermeulen M, Hijdra A, et al: Hyponatremia and cerebral infarction in patients with ruptured intracranial aneurysms: is fluid restriction harmful? Ann Neurol 17:137-140, 1985

77. Wilkinson E, Rieff J, Rekate HL, et al: Fluid, blood, and blood product management in the craniofacial patient. Pediatr Neurosurg 18:48-52, 1992

78. Yamada K, Goto A, Nagoshi H, et al: Role of brain ouabainlike compound in central nervous system-mediated natriuresis in rats. Hypertension 23:1027-1031, 1994
79. Yamaki T, Tano-oka A, Takahashi A, et al: Cerebral salt wasting syndrome distinct from the syndrome of inappropriate secretion of antidiuretic hormone (SIADH). Acta Neurochir 115:156-162, 1992

80. Zafonte RD, Mann NR: Cerebral salt wasting syndrome in brain injury patients: a potential cause of hyponatremia. Arch Phys Med Rehabil 78:540-542, 1997

Manuscript received February 18, 2004.

Accepted in final form March 2, 2004.

Address reprint requests to: William T. Couldwell, M.D., Ph.D., Department of Neurosurgery, University of Utah School of Medicine, 30 N 1900 E, Suite 3B409 Salt Lake City, Utah 84132. email: william.couldwell@hsc.utah.edu. 